Polymer Journal, Vol. 2, No. 2, pp 161-172 (1971)

\title{
Magnetic Anisotropy Effect on the Chemical Shift of Polypropylene
}

\author{
Isao ANDo and Atsuo NishiokA \\ Department of Polymer Engineering, Tokyo Institute of Technology, \\ Ookayama 2-12-1, Meguro-ku, Tokyo, Japan.
}

(Received October 2, 1970)

\begin{abstract}
The chemical shifts of the methyl, methylene, and methine protons were calculated with respect to the $3_{1}$ helix, and the (---(tt)---) and (---(tt) $\left.\left(\mathrm{g}^{\prime} \mathrm{g}^{\prime}\right)(\mathrm{tt})---\right)$ conformations of isotactic and syndiotactic polypropylenes, respectively, on the assumption that the chemical shifts are mainly due to the contribution from the $\mathrm{C}-\mathrm{C}$ bond anisotropies of the main and side chains. The latter is taken as the function of the number of successive monomer units and the deviation of the bond rotation angle $\Delta \varphi$ from the typical trans and gauche positions. It was found that the calculated chemical shifts for each group appear in the same order as the ones observed with increasing field.

KEY WORDS Chemical Shift/Magnetic Anisotropy/Polypropylene/ Conformation/NMR/
\end{abstract}

Data on high-resolution nuclear magnetic resonance (NMR) spectra of linear polymers have been considerably increased in the past ten years, but theoretical interpretations of the chemical shift in polymers have been rare because of the difficulties associated with polymer molecules. Since the first theoretical treatment of the shielding coefficient of a nucleus in a molecule was given by Ramsey, ${ }^{1,2}$ theories of the chemical shifts of simple molecules were developed quantitatively by Saika, et $a l^{3}$, Pople $^{4}$ and other investigators. However, as these treatments cannot be applied to a large molecule (especially a polymer), the interpretation of the chemical shift of the latter has to be carried out in terms of experimental findings such as the electron density, magnetic anisotropy, polar effect, solvent effect and so on. The unique character which characterizes the linear polymers from a simple low-molecular weight molecule is the diversity of conformation and configuration of the former. In other words, temperature dependence of the chemical shift of a small molecule in solution can mainly be attributed to the solvent effect, but that of a linear polymer to the change of conformation of a polymer chain. We may be able to refer to the latter as the "intrinsic" effect, which should be treated separately from the "external" solvent effect (i.e., the commonly used solvent effect).
So far in NMR spectroscopy information on the conformation of a molecule has been obtained by the spin-spin coupling, but data on the chemical shift could have significant relevance to the investigation to the conformation if the theory of the chemical shift is further developed.

With the above in mind, we studied theoretically the chemical shift of a linear polymer. In a previous paper ${ }^{5}$ we calculated the chemical shift contribution of the polar and magnetic anisotropy effects due to the side-chain carbonyl group for the methylene, and the $\alpha$-methyl protons of isotactic poly(methyl methacrylate) using $5_{1}$ and $5_{2}$ helical chain models. We obtained a result indicating that the order of the chemical shifts calculated agreed with those observed, but that the agreement between the calculated and observed chemical shifts was not satisfactory with respect to the methylene and $\alpha$-methyl protons. We believe that one of the main reasons for this discrepancy is that the magnetic anisotropy of the C-C bond was ignored in our theoretical calculation.

In this paper, we will refer to the contribution of the magnetic anisotropy $(\Delta \chi)$ of the C-C bond to the chemical shift in the helical chains for isotactic and syndiotactic polypropylenes $\left(i\right.$-PP and $s$-PP). For $i$-PP ( $3_{1}$ helix) Ritchey, et $a l .{ }^{6}$, proposed the calculated value of $0.49 \mathrm{ppm}$ for the chemical shift of the methylene protons 


\section{Ando and A. NishiokA}

by taking into account the magnetic anisotropy of the neighboring two $\mathrm{C}-\mathrm{CH}_{3}$ bonds. However we consider their calculation was inadequate for the reason that the contribution of the magnetic anisotropy of the C-C bond of a main chain to the chemical shift, which must be taken into account, was ignored and moreover the value of the chemical shift of the methylene protons in the $3_{1}$ helix they calculated is incorrect, as may be seen from our results which we will describe later. Recently Imamura, et al., ${ }^{7}$ calculated the electron densities of carbon and hydrogen atoms in $i$-PP $\left(3_{1}\right.$ helix) and $s$-PP (plannar zigzag) and interpreted qualitatively the order of the observed proton chemical shift in terms of the electron densities of carbon atoms. But the order of the ${ }^{13} \mathrm{C}$ chemical shifts is inconsistent with the order of the electron densities on the carbon atoms (i.e., the order of the ${ }^{13} \mathrm{C}$ chemical shift of $\mathrm{CH}_{2}$ and $\mathrm{CH}$ is inverse in comparison with the order of the electron densities on the carbon atoms). In spite of this fact, we believe this method has some use in the interpretation of the chemical shift.

In the following, we calculate the chemical shifts of $i$-PP and $s$-PP by using the conformations confirmed by crystallographic studies ${ }^{8}$, and conformational analysis ${ }^{9}$. In practical terms, we applied the point dipole approximation ${ }^{10}$, and adopted a value of the magnetic anisotropy of $5.5 \times 10^{-30} \mathrm{~cm}^{311}$ for the C-C bond, and the magnetic anisotropy of the $\mathrm{C}-\mathrm{H}$ bond was ignored.

\section{Method of Calculation}

The contribution of the magnetic anisotropy effect of the C-C bond to the chemical shift $\delta$ may be written as follows ${ }^{10}$

$$
\delta=\frac{\Delta \chi\left(1-3 \cos ^{2} \theta\right)}{3 r^{3}}
$$

where $\Delta \chi$ is the magnetic anisotropy, $r$ is the distance between any specified proton and the midpoint of the C-C bond and $\theta$ is the angle between the directions of $\gamma$ and the $\mathrm{C}-\mathrm{C}$ bond. We assumed that the magnetic anisotropies of the skeletal and side-chain $\left(\mathrm{C}_{-} \mathrm{CH}_{3}\right) \mathrm{C}-\mathrm{C}$ bonds are equal.

Therefore if we can obtain $\gamma$ and $\theta$, we can calculate the chemical shift $\delta$ immediately. Thus we carried out the calculation in a manner similar to that described in ref 5 (Appendix A).

Let us consider a polypropylene chain. The preferred conformations confirmed by crystallographic studies ${ }^{8}$ and conformational analysis ${ }^{9}$ for bond pair $i, i+1$ within a dyad of specified stereochemical asymetry are shown in Figure 1,
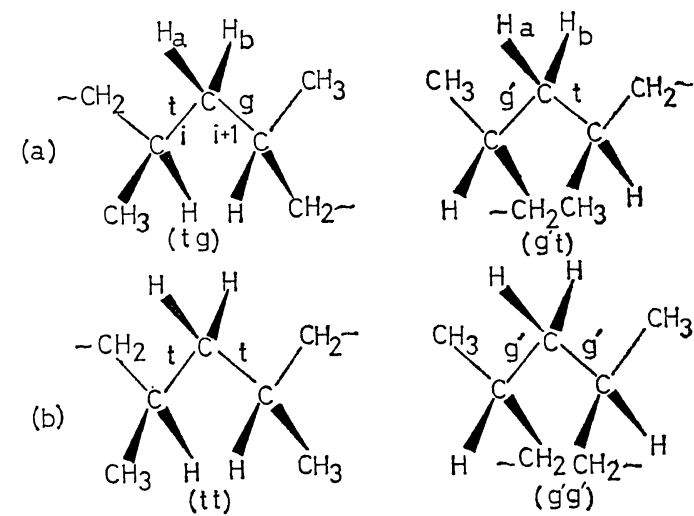

Figure 1. Preferred conformations for (a) isotactic and (b) syndiotactic dyads. The thin lines are in the plane of the drawing, while the heavy line protrude on both sides of this plane and are drawn in perspective.

where the rotational isomeric states of $t, g$ and $g^{\prime}$ are defined to occur at the rotational angles about 0,120 , and $240^{\circ}$, respectively, by a clockwise rotation around the $\mathrm{C}-\mathrm{C}$ bond, as expressed in the Newman projection. The current definition indicates that the syn and the anti protons are on the same side, and on the opposite side of the backbone to the methyl groups when the isotactic sequence assumes a planar zigzag conformation, respectively.

The bond lengths and bond angles used are shown in Table I. $^{12}$ A Hitac 5020 computer was used for all calculations.

Table I. Bond lengths and bond angles

\begin{tabular}{lc}
\hline $\mathrm{C}-\mathrm{CH}_{3}$ & $1.91(\AA)$ \\
$\mathrm{C}-\mathrm{C}$ & 1.54 \\
$\mathrm{C}-\mathrm{H}$ & 1.10 \\
$\mathrm{C}-\mathrm{C}-\mathrm{C}$ & $109.5\left(^{\circ}\right)$ \\
$\mathrm{C}-\mathrm{C}-\mathrm{CH}_{3}$ & 109.5 \\
$\mathrm{C}-\mathrm{C}-\mathrm{H}$ & 109.5 \\
\hline
\end{tabular}




\section{NUMERICAL RESULTS AND DISCUSSION}

\section{Isotactic Polypropylene}

It is known that the preferred conformations for $i$-PP are the right and left-handed $3_{1}$ helices found in the stable crystalline forms ${ }^{8}$. We calculated the chemical shift on the basis of the proposition that the helical conformation in the solid state may sometimes exist locally in solution..$^{13,14}$

We first calculated the chemical shifts for the chains of various lengths (number of monomer unit $(n)$ ) and showed the numerical results for $i$-PP and $s$-PP in Figure 2, where we took into account the contribution of the neighboring monomeric units $(n / 2)$ for these calculations and the rotational angles for the trans $\left(\varphi_{t}\right)$ and those for gauche conformations $\left(\varphi_{g}\right.$ and $\left.\varphi_{g^{\prime}}\right)$ were 0 and $\pm 120^{\circ}$, respectively. It may be seen from Figure 2 that the chemical shifts of the methylene, and methyl and methine protons approach a constant value at four and five monomer units, respectively, against the number of monomer units $(n)$. It was then conjectured that the chemical shift of a polymer chain in solution is characterized by the local part in the chain. The order of the chemical shifts of the methyl, methylene, and methine protons in $i$-PP is con-

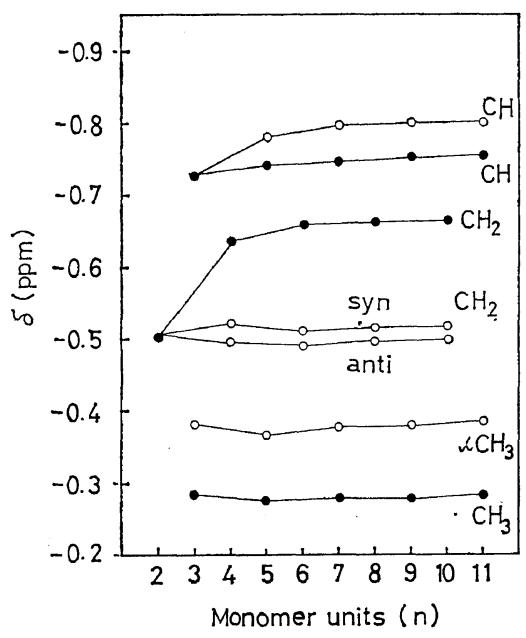

Figure 2. The chemical shift dependencies of methyl, methylene, and methine protons of isotactic (----(tg) $x_{\left.x^{----}\right)}$and syndiotactic (---(tt)---) polypropylenes on the number of monomer units: $\varphi_{t}=0^{\circ} ; \varphi_{g}=120^{\circ} ; \bigcirc$, isotactic; $\odot$, syndiotactic. sistent with the experimental order (Table II), but the order of the chemical shift between the anti and the syn protons in methylene ones is reversed. Thus we must, in calculating the chemical shift, take into account the deviation $(\Delta \varphi)$ from $0^{\circ}(t), 120^{\circ}(g)$ and $240^{\circ}\left(g^{\prime}\right)$ for the rotational angle, due to the fact ${ }^{9}$ that the

Table II. The observed chemical shifts of polypropylene

\begin{tabular}{lllll}
\hline Isotactic & \multicolumn{5}{c}{ Chemical shift, ppm } \\
\hline $\mathrm{CH}_{3}$ & $9.042^{15, \mathrm{a}}$ & $0.678^{16, \mathrm{~b}}$ & $9.08^{17, \mathrm{c}}$ & $9.0718, \mathrm{~d}$ \\
\multicolumn{1}{c}{ syn } & 9.008 & 0.650 & 9.07 & 9.05 \\
$\mathrm{CH}_{2}$ & & & & \\
\multicolumn{1}{c}{${ }_{\text {anti }}$} & 8.622 & 0.223 & 8.69 & 8.67 \\
$\mathrm{CH}$ & 8.320 & 0 & 8.31 & 8.34 \\
\hline
\end{tabular}

\begin{tabular}{llc}
\hline Syndiotactic & \multicolumn{2}{c}{ Chemical shift, ppm } \\
\hline $\mathrm{CH}_{3}$ & $9.080^{15, \mathrm{a}}$ & $9.12^{17, \mathrm{c}}$ \\
$\mathrm{CH}_{2}$ & 8.826 & 8.95 \\
$\mathrm{CH}$ & 8.320 & 8.33
\end{tabular}

a $10 \%(\mathrm{w} / \mathrm{v})$ solutions in $o$-dichlorobenzene and chlorobenzene at $168^{\circ} \mathrm{C}(60 \mathrm{MHz})$; ref signal, TMS, converted $\mathrm{Hz}$ to $\mathrm{ppm}$.

b $10 \%(\mathrm{w} / \mathrm{v})$ solution in $o$-dichlorobenzene at $140^{\circ} \mathrm{C}(60 \mathrm{MHz})$; ref signal, methine proton, converted $\mathrm{Hz}$ to ppm.

c $10 \%(\mathrm{w} / \mathrm{v})$ solution in $o$-dichlorobenzene at $140^{\circ} \mathrm{C}$ (100 MHz); ref signal, HMDS.

d $10 \%(\mathrm{w} / \mathrm{v})$ solution in $o$-dichlorobenzene at $150^{\circ} \mathrm{C}$ (220 MHz); ref signal, HMDS.

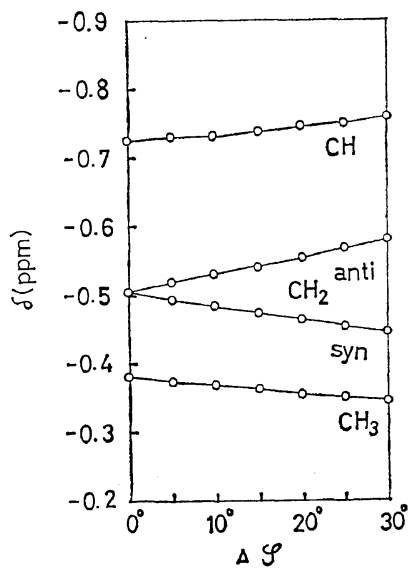

Figure 3. The chemical shift dependencies of the methyl, methylene, and methine protons of isotactic polypropylene $\left(--(t g)_{n---)}\right.$ on $\Delta \varphi(2-3$ model $)$. 


\section{Ando and A. NishiokA}

optimum location of the preferred $t g$ (or $g^{\prime} t$ ) conformation may differ by a $\Delta \varphi$ of as much as 15 or $20^{\circ}$.

We then show the chemical shift dependencies of a model (2-3 model), taking into account two and three monomer units for the methylene and methine protons (for which we wish to calculate the chemical shift) respectively and a model (10-11 model), taking into account ten and eleven monomer units in Figures 3 and 4, respectively.

It is found from Figure 3 that there is no chemical shift difference between the syn and anti protons in methylene at $\Delta \varphi=0^{\circ}$; that the difference increases with $\Delta \varphi$ (which was given according to the definition proposed by Abe, et $\left.a l .{ }^{9}\right)$, and that the order of the chemical shift of the syn and anti protons is consistent with that obtained experimentally. ${ }^{15-18}$ However, the magnitude of the calculated chemical shift difference of the syn and anti protons at $\Delta \varphi=17.5^{\circ}$ (the optimum location proposed by Abe, et al. ${ }^{9}$ ) is significantly different from the difference observed experimentally. We may therefore conclude from the results described above that the calculation proposed by Ritchey, et al. ${ }^{6}$ is incorrect.

It may be seen from Figure 4 that the chemical shift of the syn and anti protons is magnetically equivalent at $\Delta \varphi=5^{\circ}$, but not at $\Delta \varphi=0^{\circ}$;

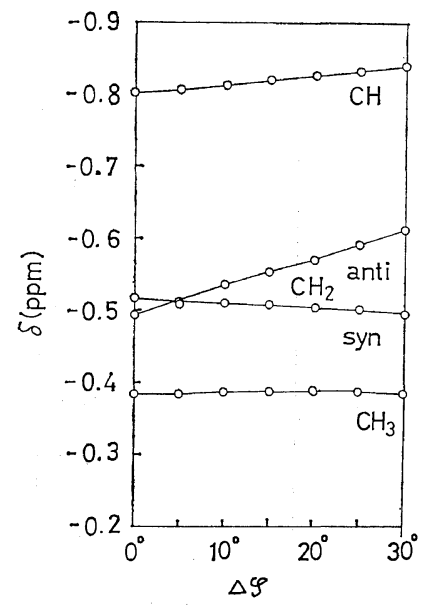

Figure 4. The chemical shift dependence of methyl, methylene, and methine protons of isotac-

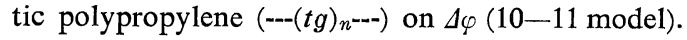

that the order of the chemical shift is opposite in to that found experimentally between 0 and $5^{\circ}$, but is consistent in the range beyond $5^{\circ}$; and that, moreover, the chemical shift difference of the syn and anti protons in the $11-12$ model is slightly less than that in 2-3 model. As described above, it was found that the calculation of the chemical shift using the $3_{1}$ helical chain model is reasonable.

The contribution of the skeletal C-C and sidechain $\mathrm{C}-\mathrm{CH}_{3}$ magnetic anisotropies to the chemical shift of $i$-PP $\left(\Delta \varphi=0^{\circ}\right)$ is shown in Figure 5. The $\mathrm{C}-\mathrm{C}$ and $\mathrm{C}-\mathrm{CH}_{3}$ bond anisotropies contribute mainly to the chemical shifts of the methylene and methine and methyl protons, respectively, because the methylene and methine, and methyl protons bond to the carbon atoms of the skeletal and side chain, respectively, and the $\mathrm{C}_{-} \mathrm{CH}_{3}$ bond anisotropy contributes slightly to shield the methylene protons at high field.

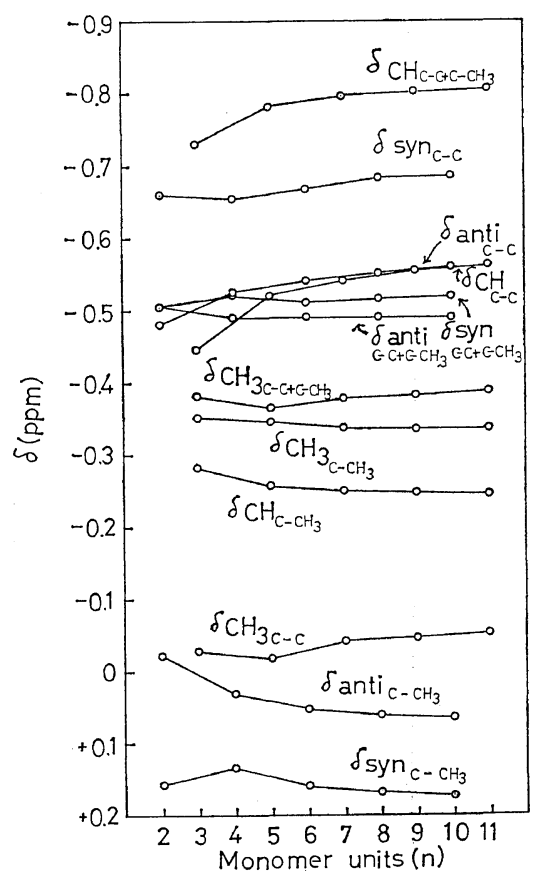

Figure 5. The contribution of the $\mathrm{C}-\mathrm{C}$ and $\mathrm{C}-\mathrm{CH}_{3}$ bond anisotropies to the chemical shifts of the methyl, methylene, and methine protons in isotactic polypropylene $\left(\Delta \varphi=0^{\circ}\right) . \quad \delta_{X_{Y}}$ is the chemical shift of the $X$ proton due to the $Y$ bond anisotropy. 
The Chemical Shift of Polypropylene

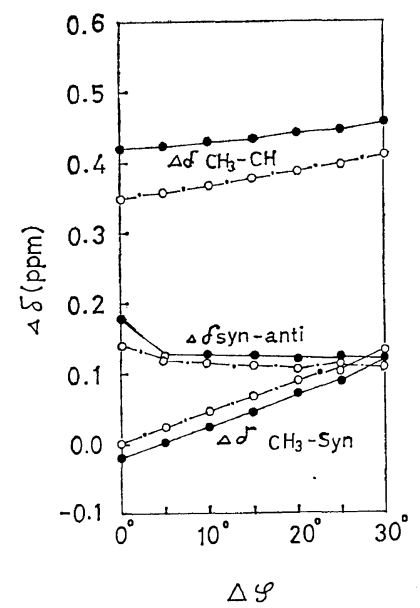

Figure 6. The chemical shift difference $\left(\delta_{\mathrm{CH}_{3}}-\delta_{\mathrm{CH}}\right.$, $\delta_{s y n}-\delta_{a n t i}$ and $\left.\delta_{\mathrm{CH}_{3}}-\delta_{\text {syn }}\right)$ dependencies of isotactic polypropylene on $\Delta \varphi: \bigcirc(-\cdot), 2-3$ model; $(-)$, 10-11 model.

The chemical shift differences between the methyl and methine protons $\left(\Delta \varphi_{\mathrm{CH}_{3}-\mathrm{CH}}\right)$, the $s y n$ and anti protons $\left(\Delta \delta_{s y n-a n t i}\right)$, and the methyl and syn proton $\left(\Delta \delta_{\mathrm{CH}_{3}-s y n}\right)$ against $\Delta \varphi$ are indicated in Figure 6. It is shown that the values of $\Delta \delta_{\mathrm{CH}_{3}-\mathrm{CH}}$ 's and $\Delta \varphi_{\text {syn-anti }}$ 's of the $2-3$ and $10-$ 11 models increase slightly with the values of $\Delta \delta ; \mathbf{C H}_{3}-s y n$ of the two models are constant when $\Delta \varphi$ is higher than $5^{\circ}$, and that these results do not agree with those obtained experimentally.

Although the values of chemical shifts of isotactic polypropylene which we calculated compare favourably, in a qualitative sense, with data obtained experimentally, they are, however, quantitatively inadequate.

\section{Syndiotactic Polypropylene}

It has been found that the preferred conformations of syndiotactic polypropylene in a crystalline state, confirmed by crystallographic studies $^{8}$ are [--- $\left.(t t)(t t)(t t)---\right]$ and [---(tt) $\left.\left(g^{\prime} g^{\prime}\right)(t t)---\right]$. We then calculated the chemical shifts of $s$-PP which has these conformations, on a similar basis to that described above.

The chemical shift $(\delta)$ on the planar zigzag conformation is shown together with the chemical shift of $i$-PP against $n$ in Figure 2. It is found that the chemical shifts of the methylene, and methyl and methine protons, approach a constant value at four and five monomer units, respectively, against $n$. These chemical shift dependencies on $n$ are similar to those of $i$-PP

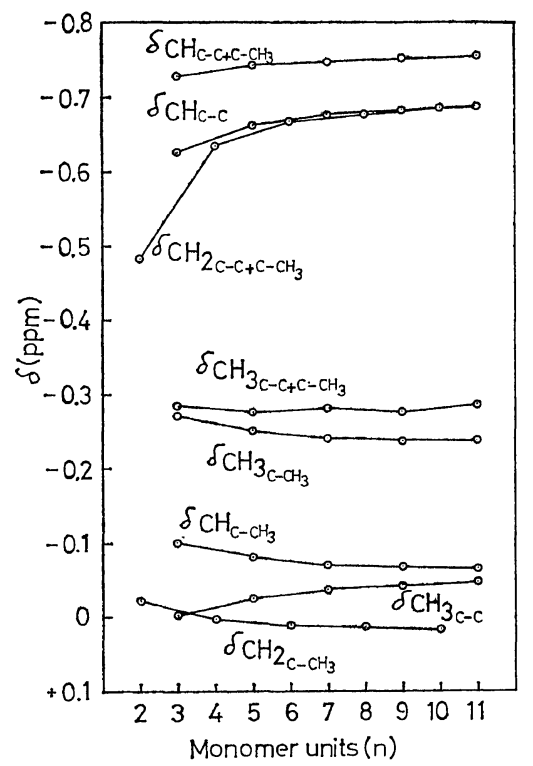

Figure 7. The contribution of the $\mathrm{C}-\mathrm{C}$ and $\mathrm{C}-\mathrm{CH}_{3}$ bond anisotropies to the chemical shifts of the methyl, methylene, and methine protons in syndiotactic polypropylene. $\delta_{X_{Y}}$ is the chemical shift of the $X$ proton due to the $Y$ bond anisotropy $\left(\Delta \varphi=0^{\circ}\right)$.

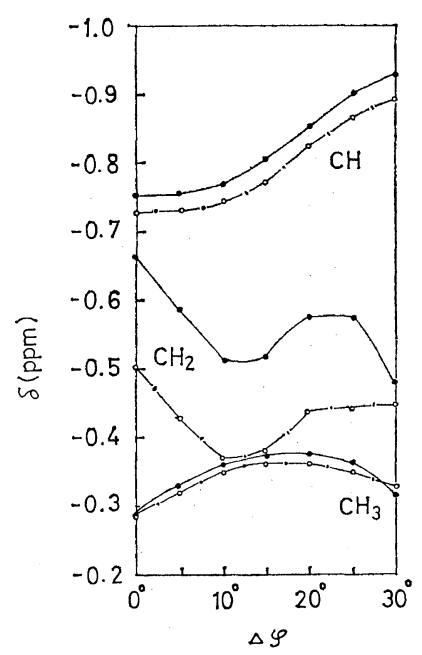

Figure 8. The chemical shift dependencies of the methyl, methylene, and methine protons of syndiotactic polypropolene (--( $\left.\mathrm{tt})_{n^{---}}\right)$on $\Delta \varphi: \bigcirc(-\cdot)$, 2-3 model; $(-), 10-11$ model. 


\section{ANDo and A. NishiokA}

except for the methylene protons. The chemical shift dependence of the methylene protons of $s$-PP on $n$ is greater in comparison with that of $i$-PP. The order of the chemical shifts of the methyl, methylene, and methine protons is consistent with the experimental order.

The contribution of the skeletal C-C and sidechain $\mathrm{C}-\mathrm{CH}_{3}$ magnetic anisotropies to the chemical shift of the planar zigzag $s$-PP $(\Delta \varphi=$ $0^{\circ}$ ) is shown in Figure 7. It is found that the $\mathrm{C}-\mathrm{C}$ and $\mathrm{C}-\mathrm{CH}_{3}$ bond anisotropies contribute mainly to the chemical shifts of the methylene and methine, and methyl protons respectively. These results are similar to $i$-PP shown in Figure 5.

The chemical shift dependence of two models (2-3 and 10-11 models) taking into account two and three monomer units, and ten and eleven monomer units on $\Delta \varphi$ for the methylene, and methyl and methine protons (for which we wish to calculate the chemical shift), respectively, is shown in Figure 8. The chemical shift dependence of the methyl, methylene and methine protons of $s$-PP on $\Delta \varphi$ is more complicated in comparison with that of $i$-PP. The chemical shift dependence of the methylene protons (10-11 model) on $\Delta \varphi$ has the minimum and maximum values at $\Delta \varphi=13$ and $20^{\circ}$ respectively, and the minimum value is given at $\Delta \varphi=13^{\circ}$ in the 10-11 model. The dependencies of the

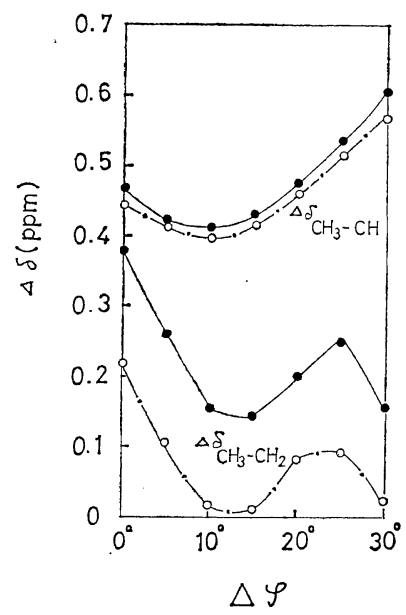

Figure 9. The chemical shift difference $\left(\Delta \delta_{\mathrm{CH}_{3}-\mathrm{CH}_{2}}\right.$ and $\left.\Delta \delta_{\mathrm{CH}_{3}-\mathrm{CH}}\right)$ dependencies of syndiotactic poly-

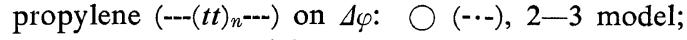
- (-), 10-11 model. methyl and methine protons on $\Delta \varphi$ have a maximum value at $\Delta \varphi=17^{\circ}$ and increase sigmoidally, respectively.

The chemical shift differences between the methyl and methylene protons $\left(\Delta \delta_{\mathrm{CH}_{3}-\mathrm{CH}_{2}}\right)$, and the methyl and methine protons $\left(\Delta \delta_{\mathrm{CH}_{3}-\mathrm{CH}}\right)$ against $\Delta \varphi$ are shown in Figure 9. It is found that the chemical shift differences $\Delta \delta_{\mathrm{CH}_{3}-\mathrm{CH}}$ and $\Delta \delta_{\mathrm{CH}_{3}-\mathrm{CH}_{2}}$ have a minimum at $\Delta \varphi=10^{\circ}$, and a minimum at $13^{\circ}$ and a maximum at $26^{\circ}$, respectively (2-3 and 10-11 models). These results are more complicated than those of $i$-PP.

The chemical shift dependence of another preferred conformation $\left(g^{\prime} g^{\prime}\right)$ on $\Delta \varphi$ is given in Figure 10. The chemical shift dependencies of the methyl, methine and methylene protons on $\Delta \varphi$ increase, decrease monotonously, and have a minimum at $\Delta \varphi=5^{\circ}$ respectively. The order of the chemical shifts of the methyl, methylene, and methine protons is consistent with the experimental order in the range of $\Delta \varphi$ calculated. However the order of the chemical shifts of the methyl, methylene, and methine protons in the 10-11 model is inconsistent with the experimental. This discrepancy is only to be expected when account is taken of the fact that a long

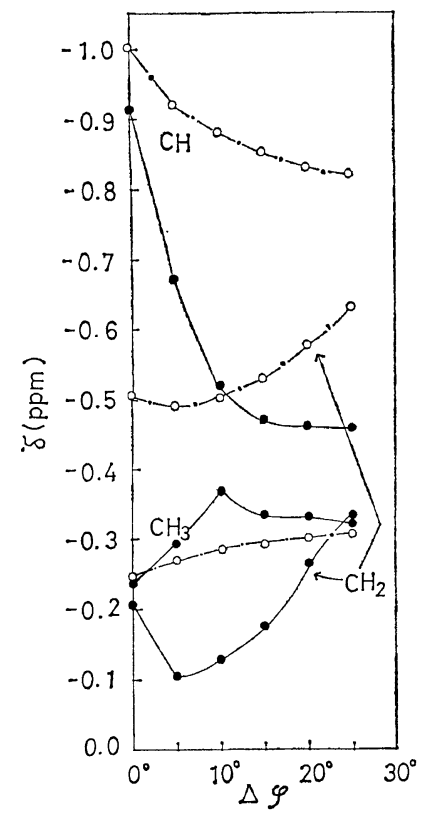

Figure 10. The chemical shift dependencies of syndiotactic polypropylene $\left(---\left(g^{\prime} g^{\prime}\right)_{n---)}\right.$ on $\Delta \varphi: \bigcirc(-\cdot-)$, 2-3 model; - (-), 10-11 model. 
sequence of the $g^{\prime} g^{\prime}$ conformation for $s$-PP cannot be sustained due to steric hindrance.

The chemical shift differences between the methyl and methylene protons $\left(\Delta \delta_{\mathrm{CH}_{3}-\mathrm{CH}_{2}}\right)$, and the methyl and methine protons $\left(\Delta \delta_{\mathrm{CH}_{3}-\mathrm{CH}}\right)$

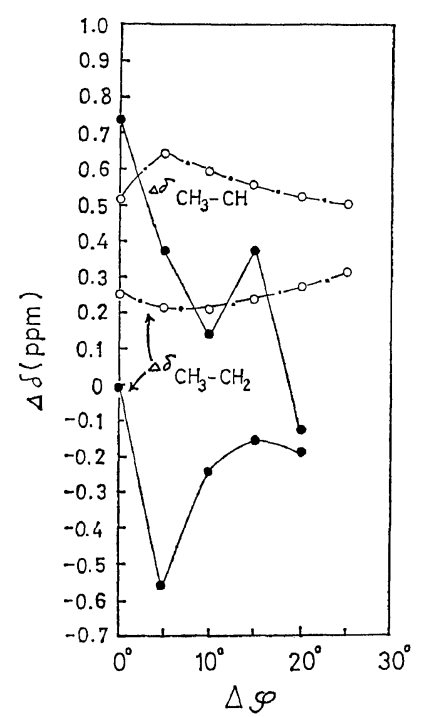

Figure 11. The chemical shift differences between the methyl and methylene protons $\left(\Delta \delta \mathrm{CH}_{3}-\mathrm{CH}_{2}\right)$, and the methyl and methine protons $\left(\Delta \delta_{\left.\mathrm{CH}_{3}-\mathrm{CH}\right)}\right.$ against $\Delta \varphi$.

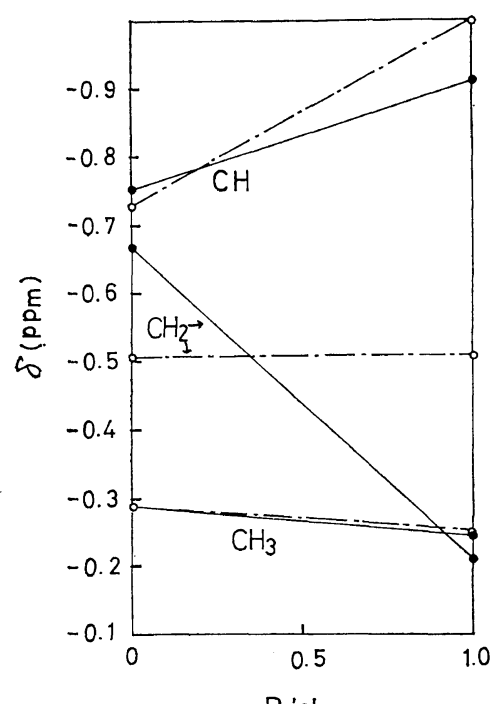

Pg'g'

Figure 12. The chemical shift dependencies of syndiotactic polypropylene on $P_{g^{\prime} g^{\prime}}\left(\Delta \varphi=0^{\circ}\right): \bigcirc(-\cdot-)$, 2-3 model; $(-), 10-11$ model. against $\Delta \varphi$ are shown in Figure 11. The chemical shift difference dependencies $\left(\Delta \delta_{\mathrm{CH}_{3}-\mathrm{CH}_{2}}\right.$ and $\left.\Delta \delta_{\mathrm{CH}_{3}-\mathrm{CH}_{2}}\right)$ of the 2-3 model are more complicated than those of the 10-11 model.

If two conformations $\left((t t)\right.$ and $\left.\left(g^{\prime} g^{\prime}\right)\right)$ in the 2-3 model of $s$-PP are assumed, the chemical shift $\delta$ may be given as ${ }^{19}$

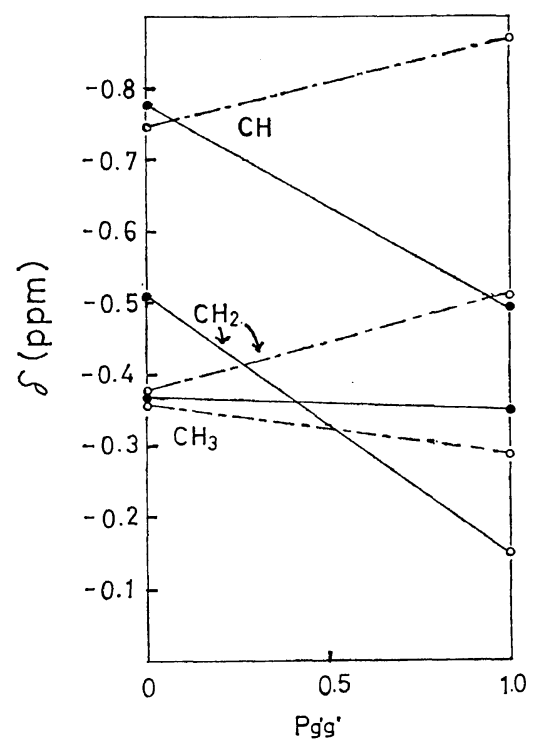

Figure 13. The chemical shift dependencies of syndiotactic polypropylene on $\boldsymbol{P}_{g^{\prime} g^{\prime}}\left(\Delta \varphi=13^{\circ}\right): \bigcirc(-\cdot)$, 2-3 model; (-), 10-11 model.

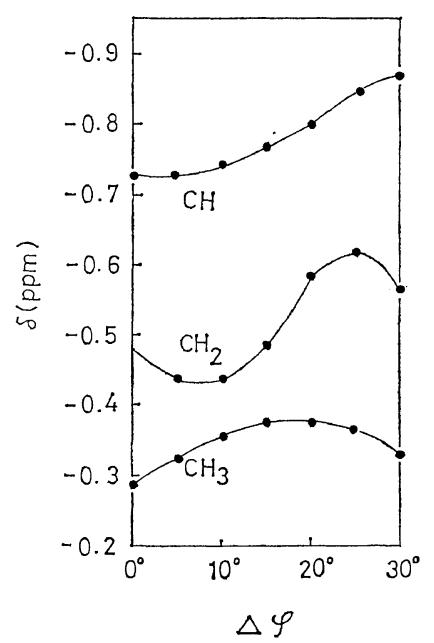

Figure 14. The chemical shift dependencies of the $(t t)\left(g^{\prime} g^{\prime}\right)(t t)$ conformation on $\Delta \varphi$. 


\section{Ando and A. NishiokA}

$$
\begin{gathered}
\delta=\boldsymbol{P}_{t t} \cdot \delta_{t t}+\boldsymbol{P}_{g^{\prime} g^{\prime}} \delta_{g^{\prime} g^{\prime}} \\
\boldsymbol{P}_{t t}+\boldsymbol{P}_{g^{\prime} g^{\prime}}=1
\end{gathered}
$$

where $P_{t t}$ and $P_{g^{\prime} g^{\prime}}$ are the fractions of the $(t t)$ and $\left(g^{\prime} g^{\prime}\right)$ conformations $s$-PP, respectively, and $\delta_{t t}$ and $\delta_{g^{\prime} g^{\prime}}$ are the chemical shifts of $s$-PP taking all the $(t t)$ and $\left(g^{\prime} g^{\prime}\right)$ conformations, respectively.

The chemical shift dependencies of $s$-PP on $P_{g^{\prime} g^{\prime}}$ at $\Delta \varphi=0^{\circ}$ and $\Delta \varphi=13^{\circ}$ are shown in Figures 12 and 13 , respectively $(2-3$ and $10-$ 11 models), but the $10-11$ model is inappropriate. From these results it was found that the values of $P_{g^{\prime} g^{\prime}}$ must be higher than 0.5 in comparison with the chemical shift of $i$-PP, as may be seen later.

We also calculated the chemical shift dependencies of the $(t t)\left(g^{\prime} g^{\prime}\right)(t t)$ conformation on $\Delta \varphi$ and showed these results in Figure 14. These tendencies are similar to those of the tt conformation (Figure 8). The order of the chemical shift calculated is consistent with the experimental one.

The chemical shift differences between the methyl and methylene protons $\left(\Delta \delta_{\mathrm{CH}_{3}-\mathrm{CH}_{2}}\right)$, and the methyl and methine protons $\left(\Delta \delta_{\mathrm{CH}_{3}}\right.$ - $\left.\mathrm{CH}\right)$ against $\Delta \varphi$ are shown in Figure 15. These tendencies are also similar to those of the $t$ conformation (Figure 9).

From the results described above it may be

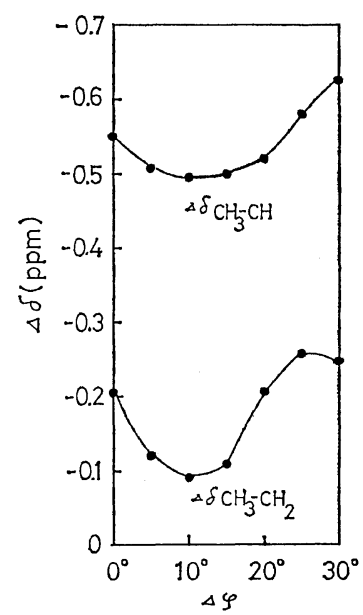

Figure 15. The chemical shift differences between

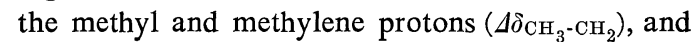
the methyl and methine protons $\left(\Delta \delta_{\mathrm{CH}_{3}} \mathrm{CH}\right)$ against $\Delta \varphi$. conjectured that the preferred conformations in solution for $s$-PP are the (---tt---) and (--- $(t t)\left(g^{\prime} g^{\prime}\right)$ $(t t)---)$ conformations (the $(t t)$ and $\left(g^{\prime} g^{\prime}\right)$ conformations for the 2-3 model).

Thus, the chemical shift of $s$-PP, as described above, may be written as follows ${ }^{19}$

$$
\begin{gathered}
\delta=\boldsymbol{P}_{t t} \delta_{t t}+\boldsymbol{P}_{(t t)\left(g^{\prime} g^{\prime}\right)(t t)} \delta_{(t t)\left(g^{\prime} g^{\prime}\right)(t t)} \\
\boldsymbol{P}_{t t}+\boldsymbol{P}_{(t t)\left(g^{\prime} g^{\prime}\right)(t t)}=1
\end{gathered}
$$

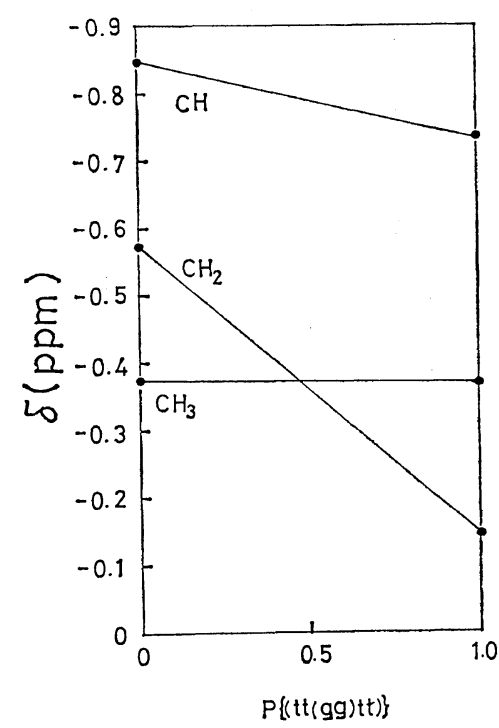

Figure 16. The chemical shift dependencies of syndiotactic polypropylene on $P(t t)\left(g^{\prime} g^{\prime}\right)(t t)$ at $\Delta \varphi=0^{\circ}$.

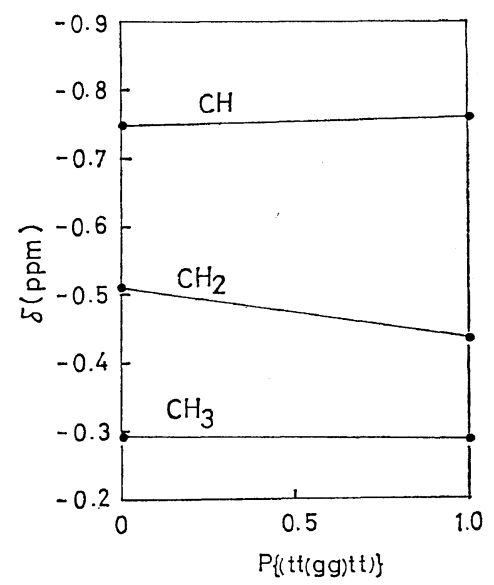

Figure 17. The chemical shift dependencies of syndiotactic polypropylene on $P(t t)\left(g^{\prime} g^{\prime}\right)(t t)$ at $\Delta \varphi=$ $13^{\circ}$. 


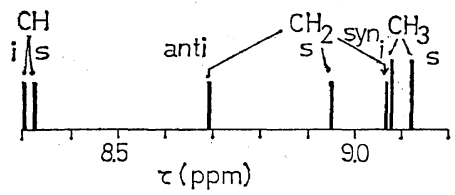

(a)

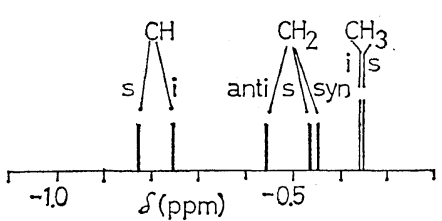

(c)

$P_{\mathrm{tt}}=1$

$\Delta \varphi=20^{\circ}$

(e)

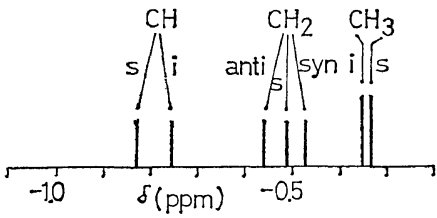

(g)

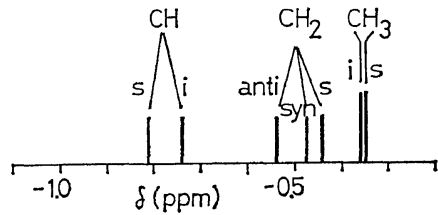

$P_{\mathrm{tt}}=0.5$

$\Delta y=13^{\circ}$

$P_{t t}=0.5$

$\Delta \varphi=20^{\circ}$

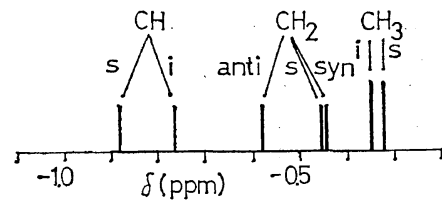

(f)

$\mathrm{Ptt}_{\mathrm{tt}}=0.5$

$\Delta \varphi=30^{\circ}$

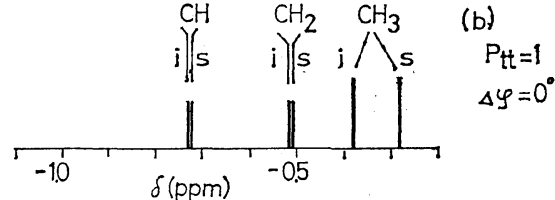

(d)

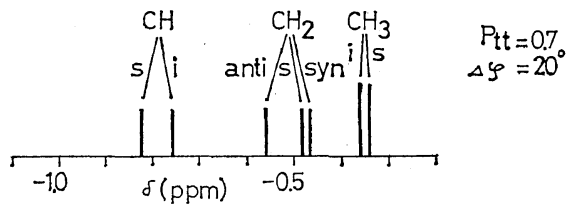

$\varphi=20^{\circ}$

$\Delta \varphi=30^{\circ}$ (i)

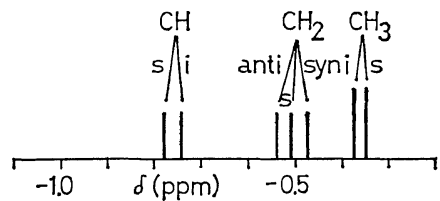

$(k)$

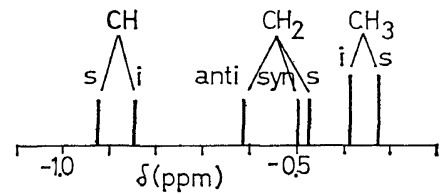

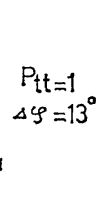

(j)

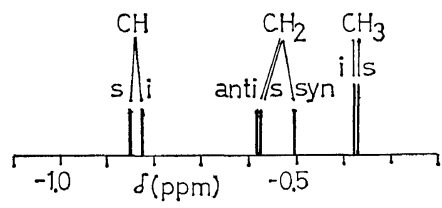

\section{$P_{\mathrm{tt}}=1$}

$\Delta \varphi=20^{\circ}$

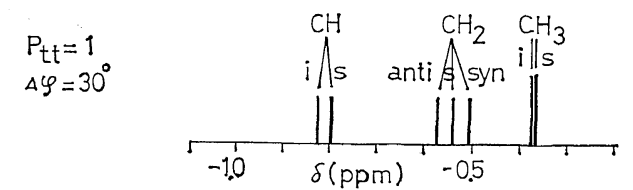

(1)

$P_{\mathrm{tt}}=0.9$ $\Delta \varphi=20^{\circ}$

Figure 18. The observed and calculated stick spectra of polypropolene: $i$, isotactic; $s$, syndiotactic; anti, anti proton; syn, syn proton: (a), observed spectrum, $10 \%$ in odichlorobenzene at $150^{\circ} \mathrm{C}(100 \mathrm{MHz})^{14}$; (b)-(g), calculated spectra of the 2-3 model (the preferred conformations are $t g$, and $t t$ and $g^{\prime} g^{\prime}$ for $i$-PP and $s$-PP respectively); $P_{t t}$ is the fraction of the $t t$ conformation for $s-\mathrm{PP}$; (h)-(q), calculated spectra of the 10-11

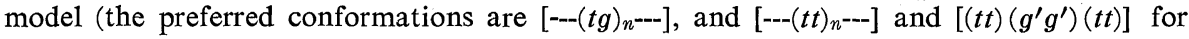

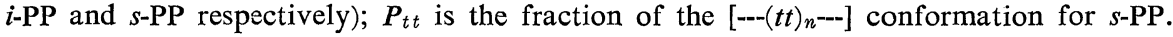


(m)

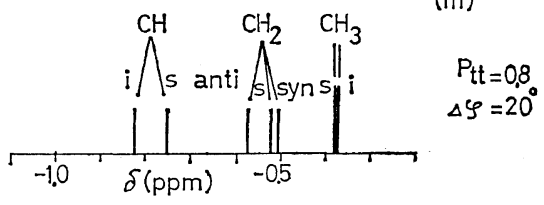

(o)

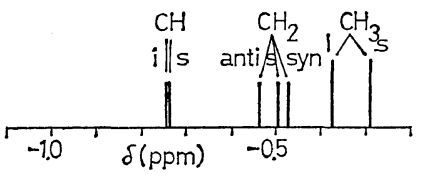

$\mathrm{P}_{\mathrm{tt}}=0.9$

$\Delta \varphi=13^{\circ}$ (n)

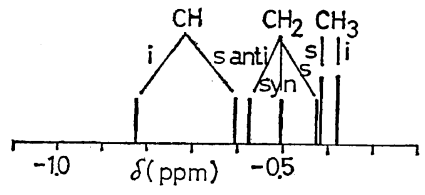

(P)

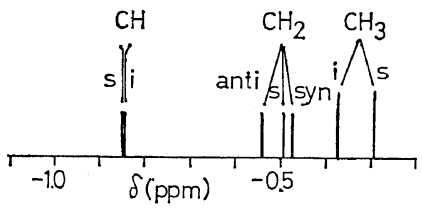

$P_{t t}=0.5$

$\Delta \varphi=20^{\circ}$

$P_{t t}=0.8$

$\Delta \varphi=13^{\circ}$

(q)

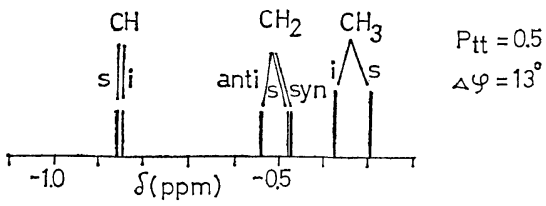

Figure 18.-continued (m)-(q).

where $\boldsymbol{P}_{(t t)\left(g^{\prime} g^{\prime}\right)(t t)}$ and $\delta_{(t t)\left(g^{\prime} g^{\prime}\right)(t t)}$ are the fraction of the $\left((t t)\left(g^{\prime} g^{\prime}\right)(t t)\right)$ conformation. The chemical shift dependencies of $s$-PP on $P_{(t t)\left(g^{\prime} g^{\prime}\right)}$ (tt) at $\Delta \varphi=0^{\circ}$ and $\Delta \varphi=13^{\circ}$ are shown in Figure 16 and 17, respectively. The chemical shift dependencies at $\Delta \varphi=0^{\circ}$ are greater than those at $\Delta \varphi=13^{\circ}$.

We discussed the chemical shift of polypropylene from the results shown in Figures 12, 13, 16, and 17, and in Figures $18 \mathrm{a}-\mathrm{q}$ compared the calculated chemical shifts of $i$-PP and $s$-PP are taken as being equal to simplify calculations. It may, however, be a reasonable assumption. The observed stick spectrum of polypropylene $^{17}$ is shown in Figure 18a. The calculated chemical shifts of polypropylene using the preferred $(t g)$, and $(t t)$ and $\left(g^{\prime} g^{\prime}\right)$ conformations for $i$-PP and $s$-PP $(2-3$ model $)$ respectively are shown in Figures $18 \mathrm{~b}-\mathrm{g}$.

The order of the chemical shift of the methine protons in the $2-3$ model of the preferred $\mathrm{tg}$ and $\mathrm{tt}$ conformations for $i$-PP and $s$-PP, respectively, as shown in Figures $18 \mathrm{~b}$ and $\mathrm{c}$, is inconsistent with the experimental order, even if the value of $\Delta \varphi$ increases. Also, the order of the chemical shifts in the $2-3$ model of the preferred $t g$, and $t t$ and $g^{\prime} g^{\prime}$ conformations of $i$-PP and $s$-PP respectively under the optimum condition $\left(P_{\mathrm{tt}}>0.5\right.$ and $\left.\Delta \varphi \approx 20^{\circ}\right)$ is inconsistent with the experimental order only for the methine proton, as shown in Figures $18 \mathrm{~d}-\mathrm{g}$. When $\boldsymbol{P}_{t t}<0.5$, moreover, the order of the chemical shift of the methylene protons is also inconsistent.

The order of the chemical shift of the methine proton in the 10-11 model of the preferred [--- $\left.(t g)_{n}---\right]$ and [--- $\left.(t t)_{n}---\right]$ for $i$-PP and $s$-PP respectively at $\Delta \varphi=0^{\circ}$ is consistent with the experimental order, but that of the methylene protons is not, as shown in Figures $18 \mathrm{~h}-\mathrm{k}$. Even if the value of $\Delta \varphi$ increases, the order of the chemical shift of the methine proton is inconsistent with the experimental one and at $\Delta \varphi=$ $30^{\circ}$, moreover, that of the methylene protons is not either.

The order of the chemical shifts in the 1011 model of the preferred [--- $\left.(t g)_{n}---\right]$, and $\left[--(t t)_{n}---\right]$ and $\left[(t t)\left(g^{\prime} g^{\prime}\right)(t t)\right]$ for $i$-PP and $s$-PP, respectively, under the optimum condition (Figures 181 and o), is consistent, but under other conditions (Figures $18 \mathrm{~m}, \mathrm{n}, \mathrm{p}$, and $\mathrm{q}$ ), it is not. At $\Delta \varphi=20^{\circ}$, and $13^{\circ}$, the order is consistent when $\boldsymbol{P}_{t t}$ is more than about 0.9. Thus the optimum proportion is about $P_{t t}=0.9$ in a range between $\Delta \varphi=10$ and $20^{\circ}$. Therefore this proportion of the $(t t)$ conformation is reasonable compared with the value $0.7 \pm 0.1$ reported by F. Heatley, et al. ${ }^{18}$ in terms of the coupling 
constant.

From the results described above, we conclude that the order of the chemical shifts of isotactic and syndiotactic polypropylenes qualitatively is consistent with the experimental order, but quantitatively it is inadequate. The reason for this quantitative inadequacy may be due to the assumption that the magnetic anisotropies of the C-C and $\mathrm{C}-\mathrm{CH}_{3}$ bonds are equal to each other; to the fact that the magnetic anisotropy of the $\mathrm{C}-\mathrm{H}$ bond is not taken into account and that the value $5.5 \times 10^{-30} \mathrm{~cm}^{311}$ of the magnetic anisotropies of the C-C and $\mathrm{C}-\mathrm{CH}_{3}$ bonds, obtained from the simple molecules, is adopted. Moreover, it is reasonable in light of results from crystallographic studies $^{8}$ and conformational analysis ${ }^{9}$ that the preferred conformations for isotactic and syndiotactic polypropylenes are $\left[---(t g)_{n}---\right]$, and $\left[---(t t)_{n}---\right]$ and $\left[(t t)\left(g^{\prime} g^{\prime}\right)(t t)\right]$, respectively, and that the optimum location for the preferred $t, g$ and $g^{\prime}$ conformations may differ by a $\Delta \varphi$ of as much as 15 or $20^{\circ}$.

Although there could be some discrepancies from the ideal straight helical chain model if we imagine the considerable flexible chain conformation of the polymer molecule in solution, it could be significant that our calculations explain the validity of the assignment of the chemical shifts of polypropylene and confirm the validity of the preferred conformations for isotactic and syndiotactic polyprepylenes.

\section{CONCLUSION}

(I) The order of the chemical shifts of the $\mathrm{CH}, \mathrm{CH}_{2}$, and $\mathrm{CH}_{3}$ protons due to the magnetic anisotropy of the C-C bond is consistent with that obtained experimentally. (II) Although there is a significant difference between the chemical shifts of two and three monomeric units the chemical shifts of three monomeric units are similar to those of long monomeric units. This tendency, however, does not contradict our experimental results. (III) The results obtained from varying the rotation angle $\Delta \varphi$ from $0^{\circ}$ show closer agreement with experimental data than in the case of the regular angle $\Delta \varphi=0^{\circ}$. (IV) The chemical shift of the syn proton in the isotactic methylene group ap- pears at higher field than in the case of the anti proton. (V) The following factors explain the quantitative disagreement between the calculated and experimental results: (a) the contribution of $\Delta \varphi_{\mathrm{C}-\mathrm{H}}$ is not taken into account; (c) relevance of McConnell's equation at short distances ( $\lesssim 1.6 \AA$ ); (d) ambiguity of the $\mathrm{CH}-\mathrm{CH}_{2}$ and C$\mathrm{CH}_{3}$ bond lengths, and (e) the fact that solvent and temperature effects are ignored.

In future quantitative studies it will be necessary to take into account these and any other factors causing results to differ from calculated values.

Acknowledgement. The authors gratefully acknowledge the helpful comments made by Dr. Naoya Nakagawa of the University of ElectroCommunications, and Dr. Akihiro Abe of the Central Research Laboratory of Showa Denko Company.

\section{APPENDIX A}

We calculated the coordinates of atoms in polypropylene as follows. Introducing local systems of coordinates linked to the bond of the chain, we can write the matrix that transforms the coordinates of a point in the $(i+1)$ th coordinate system into the (i) th coordinate system as

$\boldsymbol{T}_{i+1}(\theta, \varphi)=\left[\begin{array}{ccc}-\cos \theta \cos \varphi_{i+1} & \sin \varphi_{i+1} & \sin \theta \cos \varphi_{i+1} \\ -\cos \theta \sin \varphi_{i+1} & -\cos \varphi_{i+1} & \sin \theta \sin \varphi_{i+1} \\ \sin \theta & 0 & \cos \theta\end{array}\right]$

where $\theta$ is the angle defined by $(\theta+$ bond angle $)=$ $180^{\circ}$ and $\varphi$ the internal rotation angle. The transformation of the $(i+1)$ th coordinate $X_{i+1}$, $\left.\mathbf{Y}_{i+1}, \mathbf{Z}_{i+1}\right)$ into the $(i)$ th coordinate $\left(\mathbf{X}_{i}, \mathbf{Y}_{i}, \mathbf{Z}_{i}\right)$ is expressed as

$$
\boldsymbol{r}_{i}=T_{i+1}(\theta, \varphi) \boldsymbol{r}_{i+1}
$$

where

$$
\boldsymbol{r}_{i}=\left[\begin{array}{c}
X_{i} \\
Y_{i} \\
Z_{i}
\end{array}\right]
$$

Thus we can transform the coordinates of atoms into the coordinate system linked to the bond $(i=1)$ and determine the coordinates of each atom in polypropylene. 


\section{REFERENCES}

1. N.F. Ramsey, Phys. Rev., 78, 699 (1950).

2. N.F. Ramsey, Phys. Rev., 86, 243 (1952).

3. A. Saika and C.P. Slichter, J. Chem. Phys., 22, 26 (1954).

4. J. A. Pople, Proc. Roy. Soc., A239, 541, 550 (1957).

5. I. Ando and A. Nishioka, Polymer J., 1, 288 (1970).

6. W.M. Ritchey and F.J. Knoll, J. Polym. Sci., Part B, 4, 853 (1966).

7. A. Imamura, Y. Fujita, and C. Nagata, Preprint, Symposium on Molecular Structure, The Chemical Society of Japan, Fukuoka, 1969, p 211.

8. G. Natta and P. Corradini, J. Polym. Sci., 20, 251 (1956); G. Natta, I. Pasquon, P. Corradini, M. Peraldo, M. Pegoraro, and A. Zambelli, Atti Accad. Nazl. Lincei Rend. Classe Sci. Fis. Mat. Nat., 28, 539 (1960); G. Natta, Makromol. Chem., 35, 93 (1960); G. Natta, M. Peraldo, and G. Allegra, Makromol. Chem., 75, 215 (1964).
9. N.P. Borisova and T.M. Birshtein, Vysokomol. Soedin., 5, 279 (1963).

P. J. Flory, J. E. Mark, and A. Abe. J. Amer. Chem. Soc., 88, 639 (1966).

10. H. M. McConnell, J. Chem. Phys., 27, 226(1957).

11. A. A. Bothner-By and C. Naar-Colin, Ann. $N$. Y. Acad. Sci., 70, 833 (1958).

12. L. Pauling, "Nature of Chemical Bond," Cornell University Press, Iowa, 1960.

13. S. Mizushima and T. Shimanouchi, J. Amer. Chem. Soc., 86, 3521 (1964).

14. F. A. Bovey, F.P. Hood III, E. W. Anderson, and L. C. Snyder, J. Chem. Phys., 42, 3900 (1965).

15. W.C. Tincher, Makromol, Chem., 85, 34 (1964).

16. Y. Kato and A. Nishioka, Bull. Chem. Soc. Japan, 37, 1622 (1964).

17. J.C. Woodbery, J. Polym. Sci., Part B 2, 315 (1964).

18. F. Heatly, R. Salovey, and F.A. Bovey, Macromolecules, 2, 619 (1969).

19. H.S. Gutowsky, J. Chem. Phys., 37, 2196(1962). 